\title{
On a generalized approach to the linear stability of spatially nonuniform thin film flows
}

\author{
Jeffrey M. Davis and Sandra M. Troian ${ }^{\text {a) }}$ \\ Department of Chemical Engineering, Princeton University, Princeton, New Jersey 08544-5263
}

(Received 9 January 2002; accepted 19 January 2003; published 4 April 2003)

\begin{abstract}
The presence of a deformable free surface in thin films driven to spread by body or shear forces gives rise to base states that are spatially nonuniform. This nonuniformity produces linearized disturbance operators that are non-normal and an eigenvalue spectrum that does not necessarily predict stability behavior. The falling film provides a simple example for demonstrating a more generalized, rigorous nonmodal approach to linear stability for free surface flows. Calculations of the pseudospectra and maximum disturbance amplification in this system, however, reveal weak effects of non-normality and transient growth such that the modal growth rate is rapidly recovered. Subdominant modes contribute little energy to the leading eigenvector because the oscillatory behavior is rapidly damped by surface tension. Generalization of these results to numerous other lubrication flows involving surface tension suggests similarly weak non-normality and transient growth. (C) 2003 American Institute of Physics. [DOI: 10.1063/1.1564094]
\end{abstract}

The linear stability of thin liquid films driven to spread by shear or body forces has been studied extensively during the past decade. ${ }^{1-4}$ Prior studies have used classical modal analysis to determine the transverse wavelength and growth rate of the most unstable mode. Such an analysis implicitly assumes the linearized operator is normal and the eigenvectors orthogonal, but the presence of a deformable free surface gives rise to base state traveling wave solutions that are spatially nonuniform. This spatial inhomogeneity produces nonnormal disturbance operators. ${ }^{5}$ It is well known that the eigenvalue spectrum of highly non-normal systems may not be physically determinant. ${ }^{6}$

The presence of non-normal operators in problems involving spreading films was first explored in the context of gravitationally driven flows. ${ }^{7,8}$ To relieve the usual stress singularity at moving contact lines, the base state film profile was patched onto a flat, infinite precursor layer. ${ }^{9}$ Disturbances localized near the initial contact line were shown to undergo magnification by over two orders of magnitude. The amplification ratio, however, was not quantified by the 2-norm of the operator exponential normally associated with energy production ${ }^{10}$ but instead by the $\infty$-norm of an evolved (particular) initial disturbance. The more generalized approach to linear stability for non-normal autonomous operators described below provides a rigorous upper bound on disturbance amplification at all times by identifying the optimal disturbances from the singular value decomposition (SVD) of the propagator. ${ }^{11}$ In addition, there are alternative contact line models that can be used to study the influence of boundary conditions on the level of transient growth. This present work, which revisits the falling film using a slip boundary condition, implements a more generalized framework for examining the linear stability of thin film flows

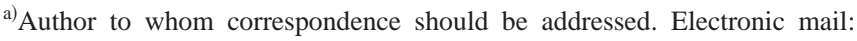
stroian@princeton.edu
}

involving free surfaces. While this work considers the effects of perturbations to the height profile of the film, other types of disturbances are possible. For example, the effect of sinusoidal perturbations to the slip coefficient has recently been investigated. $^{12}$

The well-known stress singularity associated with moving contact lines ${ }^{13}$ can be alleviated by allowing for slip at the liquid-solid interface. The Greenspan model, ${ }^{14}$ which allows adjustment of the contact angle slope, describes either partially wetting or completely wetting liquids. For appropriate choices of the slip coefficient and contact slope, it can reproduce the base state profiles and dispersion curves found with a precursor model. ${ }^{1,7}$ It can also be used to examine configurations with large capillary ridges that are highly susceptible to frontal breakup. The governing lubrication equation for the evolution of the film thickness using the Greenspan condition was first derived by Spaid and Homsy. ${ }^{2}$ Inclusion of hydrostatic pressure is straightforward, so only the final result is given below. A viscous liquid film of density $\rho$, viscosity $\mu$, surface tension $\gamma$, and characteristic thickness $h_{c}$ flows down a substrate inclined from the horizontal by an angle $\theta$. The coordinate axis $\hat{x}$ points in the uphill direction, $\hat{y}$ lies in the plane and normal to $\hat{x}$, and $\hat{z}$ lies normal to the inclined plane. The dimensionless evolution equation is given by

$$
h_{t}-\left(h^{3}+\alpha h\right)_{\chi}+\nabla \cdot\left[\left(h^{3}+\alpha h\right) \nabla\left(\nabla^{2} h-D h\right)\right]=0,
$$

where $\nabla=\hat{\chi} \partial_{\chi}+\hat{\zeta} \partial_{\zeta}$. The coefficient of the hydrostatic pressure is given by $D(\theta)=(3 C a)^{1 / 3} \cot (\theta)$, where $C a$ $=\mu U_{c} / \gamma$ is the capillary number and $U_{c}$ $=\left(\rho g \sin (\theta) h_{c}^{2}\right) /(3 \mu)$ is the characteristic speed of the falling film in the absence of hydrostatic pressure. The scalings used in Eq. (1) are $(\chi, \zeta)=(x, y) / l, h=\widetilde{h} / h_{c}, \omega=z / h_{c}$, and $t$ $=\tilde{t} U_{c} / l$, where dimensional variables are denoted by a tilde and $l=h_{c} /(3 \mathrm{Ca})^{1 / 3}$ represents the length scale for the inner region of the flow. The slip condition relates the liquid speed 


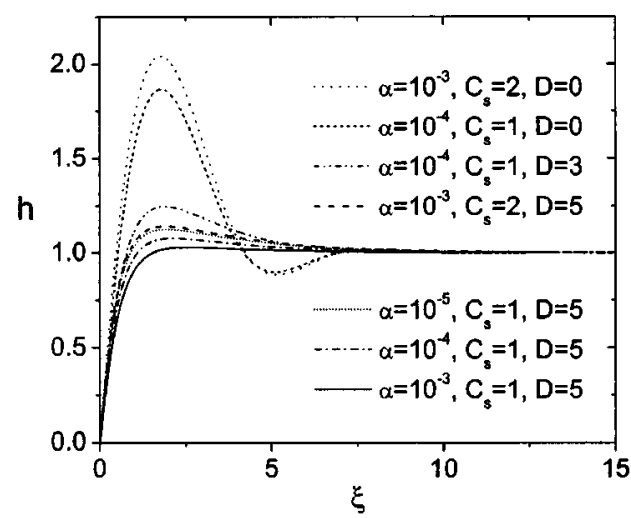

(a)

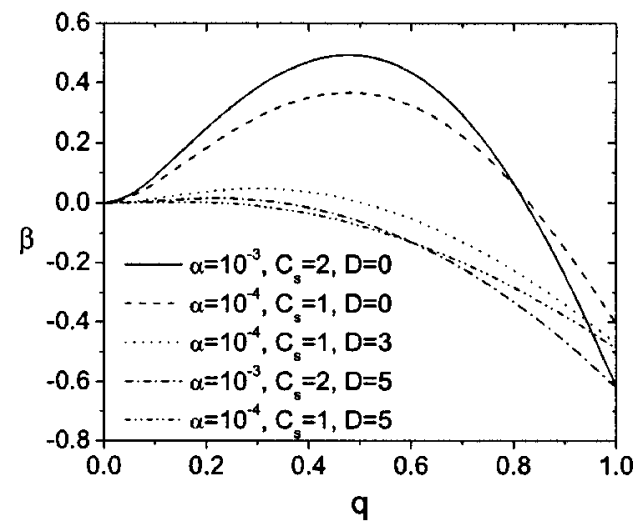

(b)

FIG. 1. Base states (a) and dispersion curves (b) for selected values of the slip parameter $\alpha$, contact slope $C_{s}$, and coefficient of the hydrostatic pressure $D$.

at the solid surface to the local shear stress through the slip coefficient $\alpha$ according to the dimensionless relation

$$
\left.v\right|_{\omega=0}=\left.\frac{\alpha}{3 h} \frac{\partial v}{\partial \omega}\right|_{\omega=0} .
$$

This system can be viewed from a reference frame moving at a constant dimensionless speed $c$ by introducing the change of variables $\xi=\chi+c t$, where $\xi=0$ denotes the position of the (unperturbed) contact line. Equation (1) is subject to the boundary conditions $h(\xi=0)=0, h_{\xi}(\xi=0)=C_{s}, h(\xi \rightarrow \infty)$ $=1$, and $h_{\xi \xi \xi}(\xi \rightarrow \infty)=0$. The parameter $C_{s}$ denotes the contact slope at $\xi=0$. Substitution of the form $h=h_{0}(\xi)$ in Eq. (1) leads to a traveling wave base state solution where $c$ $=1+\alpha$ is the wave speed. Integrating the resulting ODE once and applying the boundary conditions yields

$$
h_{0 \xi \xi \xi}=1-\frac{1+\alpha}{h_{0}^{2}+\alpha}+D h_{0 \xi} .
$$

Equation (3) was solved using a standard shooting scheme with a fourth-order Runge-Kutta method and an error tolerance set to $10^{-8}$. The numerical solution is shown in Fig. 1(a) for several values of $\alpha, C_{s}$, and $D .{ }^{15}$ The capillary ridge near the contact line is more pronounced for smaller values of the slip coefficient or larger values of the contact slope, while larger values of $D$ have a suppressive effect. Since for finite liquid volumes the characteristic film thickness decreases in time ${ }^{1}$ according to $h_{c} \sim t^{-1 / 3}$, the parameter $D$ slowly decreases as the film spreads downslope.

Equation (1) is perturbed about the traveling wave solution by imposition of two dimensional disturbances of the form $G(\xi, t) \exp (i q \zeta)$. The shape of the streamwise disturbance is governed by

$$
\begin{aligned}
\frac{\partial G}{\partial t}= & {\left[\left(D h_{0 \xi \xi}-h_{0 \xi \xi \xi \xi}\right)\left(\alpha+3 h_{0}^{2}\right)-q^{2} h_{0}\left(D+q^{2}\right)(\alpha\right.} \\
& \left.\left.+h_{0}^{2}\right)+6 h_{0} h_{0 \xi}\left(1-h_{0 \xi \xi \xi}+6 D h_{0 \xi}\right)\right] G+[(\alpha \\
& \left.\left.+3 h_{0}^{2}\right)\left(1-h_{0 \xi \xi \xi}+h_{0 \xi}\left(q^{2}+2 D\right)\right)-(1+\alpha)\right] G_{\xi} \\
& +\left[h_{0}\left(D+2 q^{2}\right)\left(\alpha+h_{0}^{2}\right)\right] G_{\xi \xi}+\left[-h_{0 \xi}\left(3 h_{0}^{2}\right.\right. \\
& +\alpha)] G_{\xi \xi \xi}+\left[-h_{0}\left(\alpha+h_{0}^{2}\right)\right] G_{\xi \xi \xi \xi},
\end{aligned}
$$

subject to the decay conditions $G, G_{\xi} \rightarrow 0$ as $\xi \rightarrow \infty$. At $\xi=0$, the boundary conditions are

$$
h_{0 \xi \xi} G-h_{0 \xi} G_{\xi}=0,
$$

which is derived from a Taylor series expansion of the base state about the (perturbed) contact line, and

$$
G_{t}+\alpha h_{0 \xi} G_{\xi \xi \xi}-\alpha\left(D+q^{2}\right) h_{0 \xi} G_{\xi}=0,
$$

which ensures that continuity is satisfied at the contact line. ${ }^{2}$ Spatial discretization of Eq. (4) using a central difference scheme yields the system of equations

$$
\frac{\partial \boldsymbol{G}}{\partial t}=\mathbf{A}(\xi) \boldsymbol{G}
$$

The linear autonomous matrix $\mathbf{A}$ acting on the state of the system $G$ contains the discretized elements; the spatial boundary conditions determine the entries in the first and last several rows. The number of grid points used to determine the discretized elements comprising matrix $\mathbf{A}$ ranged from 2000 to 4500 points. The long time behavior of solutions to Eq. (4) as $t \rightarrow \infty$ is determined from the exponential form $G(\xi, t)=H(\xi) \exp (\beta t)$, where $\beta$ denotes the disturbance growth rate. The eigenvalues and eigenfunctions of $\mathbf{A}$ were calculated using the eig function in MATLAB 5.3. ${ }^{16}$ Plots of the dispersion curves are shown in Fig. 1(b). The increasing hydrostatic pressure at low inclination angles has a strong stabilizing effect on the flow and shifts the most unstable disturbance to longer wavelengths. For sufficiently large $D$ and $\alpha$, the film becomes linearly stable to disturbances of all wavenumbers $q$.

Since $\mathbf{A}$ is non-normal, conclusions about stability derived from traditional modal analysis can prove inaccurate because the eigenvalue spectrum describes the amplification behavior of infinitesimal disturbances only in the asymptotic limit $t \rightarrow \infty$. Even when the real parts of all eigenvalues are nonpositive, significant transient growth can induce nonlinear effects and instability. For eigenvalues with positive real parts, transient amplification can still cause instability with wavenumbers and growth rates at odds with modal results. Investigations of the linear stability of disturbances governed 


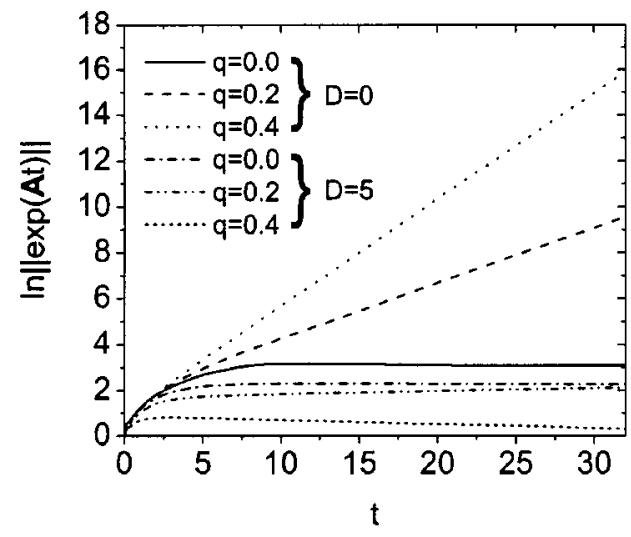

(a)

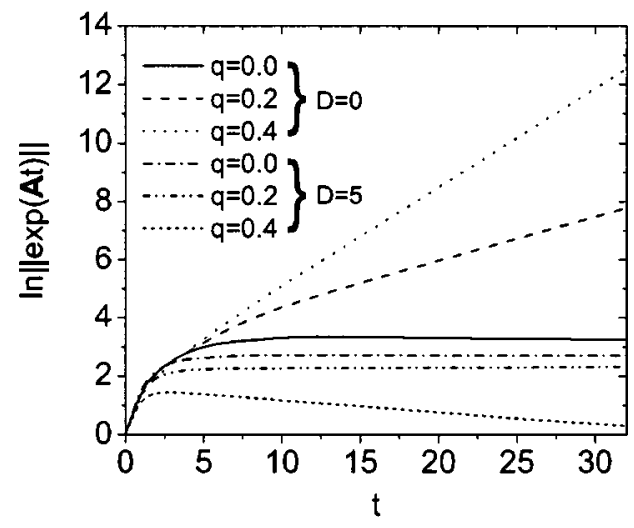

(b)

FIG. 2. Maximum attainable disturbance amplification for two selected base flow profiles with $D=0$ and $D=5$ : (a) $\alpha=10^{-3}$ and $C_{s}=2$; (b) $\alpha=10^{-4}$ and $C_{s}=1$.

by non-normal operators must therefore proceed beyond simple modal analysis to ensure the physical relevance of the results. A detailed analysis of the effects of non-normality on flow stability can be found in Ref. 11. Extension of these concepts to liquid films sheared by thermocapillary forces has also been investigated. ${ }^{17,18}$ The interested reader will find there a more in-depth treatment of the maximum transient amplification as well as the shape of optimal initial disturbances for different contact line models.

The general solution to Eq. (7) is given by $\boldsymbol{G}$ $=\exp (\mathbf{A} t) \boldsymbol{G}_{0}$, where $\boldsymbol{G}_{0}(\xi)$ represents the initial state of the system. The maximum amplification, $\|\exp (\mathbf{A} t)\|_{2}$ (where the subscript 2 denotes the Euclidean or so-called 2-norm), for selected wave numbers $q$ is shown in Fig. 2. These curves represent the envelope corresponding to the amplification of different initial conditions, maximized over all possible initial conditions. The particular initial condition that leads to the largest overall amplification can be obtained from the $\mathrm{SVD}$ of $\exp (\mathbf{A} t)$. Even for small values of the slip coefficient $\alpha$, where there exists a significant capillary ridge in the base flow, the non-modal amplification is no larger than one order of magnitude. This growth is further diminished as the hydrostatic component of the pressure $(D)$ increases. Interest-

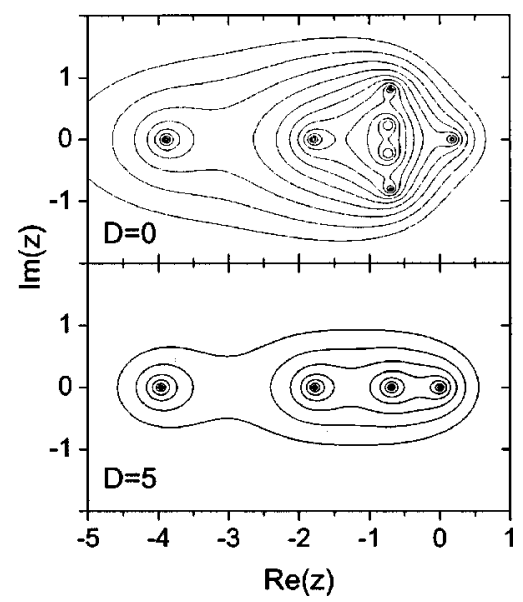

FIG. 3. Comparison of the pseudospectra for $\alpha=10^{-4}$ and $q=0.20$ with $D=0$ (top) and $D=5$ (bottom). Contours are shown for $\epsilon=10^{-1}$, $10^{-1.25}, \ldots, 10^{-3.5}$.

ingly, the extent of transient amplification is more strongly influenced by the magnitude of the slip coefficient than by the contact angle slope. In all cases, the plots of $\|\exp (\mathbf{A} t)\|_{2}$ vs $t$ demonstrate that the exponential growth rate predicted by eigenvalue analysis is rapidly attained. Additional transient growth studies conducted on thermocapillary driven films show a similarly weak nonmodal response no matter which contact line model is used. ${ }^{17,18}$ The level of transient growth produced by random disturbances in an actual experiment will be less than that predicted here since physical disturbances are not necessarily optimal.

The extent to which hydrostatic pressure diminishes the level of disturbance amplification can be examined by calculating the magnitude of the resolvent, $(z \mathbf{I}-\mathbf{A})^{-1}$ (where $\mathbf{I}$ is the identity matrix) for a range of $z \in \mathrm{C}$. For each $\epsilon \geqslant 0$, the $\epsilon$-pseudospectrum of $\mathbf{A}$ is defined as ${ }^{19}$

$$
\Lambda_{\boldsymbol{\epsilon}}(\mathbf{A})=\left\{z \in \mathrm{C}:\left\|(z \mathbf{I}-\mathbf{A})^{-1}\right\| \geqslant \epsilon^{-1}\right\} .
$$

If $\mathbf{A}$ is a normal operator, $\Lambda_{\epsilon}(\mathbf{A})$ is the union of discs formed by the set of points in $\mathrm{C}$ within a distance $\epsilon$ of the spectrum of $\mathbf{A}$. The $\epsilon$-pseudospectrum can be much larger if $\mathbf{A}$ is nonnormal. Plots of $\Lambda_{\epsilon}(\mathbf{A})$ depict the extent of non-normality and therefore the physical relevance of its eigenvalue spectrum. The pseudospectra were calculated using the Pseudospectra GUI $^{20}$ for MATLAB.

The pseudospectra near the leading eigenvalues are plotted in Fig. 3 for $\alpha=10^{-4}$ and $q=0.20$. Contours are shown for $\epsilon=10^{-1}, 10^{-1.25}, \ldots, 10^{-3.5}$. The $\epsilon=10^{-3.5}$ contours are visible in the upper plot on an $O(1)$ scale, while only the $\epsilon=10^{-2.25}$ contours are visible in the lower plot, demonstrating that the non-normality of the linearized disturbance operator decreases as $D$ increases. Different choices for the slip coefficient and wavenumber produce qualitatively similar results; the extent of non-normality diminishes as $\alpha$ and $q$ increase. The pseudospectra demonstrate mild non-normality since each $\epsilon$-pseudospectral contour exceeds the spectrum by a distance only slightly greater than $\epsilon \cdot{ }^{19}$ When combined with the plots of $\ln \|\exp (\mathbf{A} t)\|$ vs $t$ shown in Fig. 2, these results indicate that the leading eigenvalue is therefore physically determinant in each case. 
The generalized stability analysis applied to a falling film subject to a Greenspan slip model reveals less than one order of magnitude of nonmodal disturbance amplification at any inclination angle for the range of slip coefficients and contact slopes studies. This range of values encompasses configurations whose base state profiles and dispersion curves corresponding to the most unstable mode overlap entirely with those computed instead from a flat precursor model. While the magnitude of the slip coefficient, $\alpha$, influences the level of transient amplification, the contact slope, $C_{s}$, is found to have little effect. Even for the smallest slip coefficient used, the level of amplification is insufficient to induce nonlinear effects since an initial perturbation amplitude of order $\alpha$, which determines the characteristic microscopic length scale, is never amplified to an $O(1)$ quantity corresponding to the characteristic film thickness $h_{c}$. Furthermore, the time interval for transient amplification, as evident in Fig. 2, is remarkably short and insufficient to sustain response on a timescale exceeding the characteristic convective time scale. The $\epsilon$-pseudospectra confirm the small degree of non-normality in this system. These contours establish that the leading eigenvalue found from modal analysis is physically determinant. These results taken together strongly suggest that any fingering instability that occurs at small inclination angles is caused by an unstable eigenvalue and not by a transient growth mechanism induced by disturbances to the film. This conclusion is consistent with the explanation of fingering proposed by $\mathrm{Ye}$ and Chang, ${ }^{21}$ which predicts the onset of the fingering instability via spectral analysis.

The generalized method described above provides a simple but rigorous method for probing the linear stability of many spatially nonuniform thin film flows. This method, which can monitor a system's response to perturbations at any time, recovers the exact leading eigenvalue in the limit $t \rightarrow \infty$. It not only provides a temporal history of the system's response to perturbations, but also identifies the optimal perturbations that undergo maximum amplification, thereby removing any uncertainty as to which type of disturbance causes maximum destabilization. Since many driven film problems have been shown to undergo the same fingering instability as falling films and since the governing evolution equations in the inner region comprise the same balance of forces (i.e., capillary, viscous, and driving force), it is anticipated that such problems will display negligible transient growth as quantified by $\|\exp (\mathbf{A} t)\|_{2}$. Significant non-modal growth is likely absent because the angles between the dominant mode and the other eigenvectors are reasonably large $\left(>63^{\circ}\right.$ in the system studied here). This lack of "interference" from nearby modes may be due to the fact that the spatial variations of the base state are confined to a small capillary region near the moving contact line. As a result, little energy from the subdominant modes is transferred to the leading eigenvector before these more oscillatory modes are strongly damped by surface tension, which plays such a critical role in free surface lubrication flows.

\section{ACKNOWLEDGMENTS}

The authors gratefully acknowledge financial support from an NDSEG Fellowship from the Department of Defense (J.M.D.), the National Science Foundation XYZ on a Chip program (CTS-0088774), and a Princeton University MRSEC Grant (DMR-9809843).

${ }^{1}$ S. M. Troian, E. Herbolzheimer, S. A. Safran, and J. F. Joanny, "Fingering instabilities of driven spreading films," Europhys. Lett. 10, 25 (1989).

${ }^{2}$ M. A. Spaid and G. M. Homsy, "Stability of Newtonian and viscoelastic dynamic contact lines," Phys. Fluids 8, 460 (1996).

${ }^{3}$ D. E. Kataoka and S. M. Troian, "A theoretical study of instabilities at the advancing front of thermally driven coating films," J. Colloid Interface Sci. 192, 350 (1997).

${ }^{4}$ D. E. Kataoka and S. M. Troian, "Stabilizing the advancing front of thermally driven coating films," J. Colloid Interface Sci. 203, 335 (1998).

${ }^{5} \mathrm{~A}$ normal operator commutes with its adjoint. The evolution equation for free surface films, like the falling film described by Eq. (1), contains derivative terms that when linearized produce differential operators $\mathscr{L}$ that are not self-adjoint: i.e., $\langle\mathscr{L} u, v\rangle=\int_{-\infty}^{+\infty} v^{\dagger} \mathscr{L} u d \xi \neq\langle u, \mathscr{L} v\rangle$ $=\int_{-\infty}^{+\infty} u^{\dagger} \mathscr{L} v d \xi$. This non-normality is caused by the spatial dependence of the base state.

${ }^{6}$ L. N. Trefethen and D. Bau III, Numerical Linear Algebra (SIAM, Philadelphia, 1997).

${ }^{7}$ A. L. Bertozzi and M. P. Brenner, "Linear stability and transient growth in driven contact lines," Phys. Fluids 9, 530 (1997).

${ }^{8}$ L. Kondic and A. L. Bertozzi, "Nonlinear dynamics and transient growth of driven contact lines," Phys. Fluids 11, 3560 (1999).

${ }^{9}$ V. G. Friz, "Über den dynamischen randwinkel im fall der vollständigen benetzung," Z. Angew. Phys. 19, 374 (1965).

${ }^{10}$ P. J. Schmid and D. S. Henningson, Stability and Transition in Shear Flows (Springer Verlag, New York, 2001).

${ }^{11}$ B. F. Farrell and P. J. Ioannou, "Generalized stability theory. Part 1: Autonomous operators," J. Atmos. Sci. 53, 2025 (1996).

${ }^{12}$ K.-H. Hoffmann, B. Wagner, and A. Munch, "On the generation and spreading of 'finger' instabilities in film coating processes," in High Performance Scientific and Engineering Computing, Vol. 8 of Lecture Notes in Computational Science and Engineering, edited by H.-J. Bungartz, F. Durst, and C. Zenger (Springer-Verlag, Berlin, 1999), pp. 245-254.

${ }^{13}$ P. G. deGennes, "Wetting: Statics and dynamics," Rev. Mod. Phys. 57, 827 (1985).

${ }^{14} \mathrm{H}$. Greenspan, "On the motion of a small viscous droplet that wets a surface,” J. Fluid Mech. 84, 125 (1978).

${ }^{15}$ This study explored the range $\alpha \in\left[10^{-5}, 10^{-3}\right], C_{s} \in[0.1,2]$, and $D$ $\in[0,5]$. If the slip coefficient $\alpha$ in this model is set equal to the dimensionless precursor film thicknesses $b$ used in the precursor film model, then for an appropriate choice of $C_{s}$, the base state profiles and the corresponding dispersion curves can be made to overlap entirely.

${ }^{16}$ MATLAB 5.3, The Math Works, Inc., Natick, MA, 1999.

${ }^{17}$ J. M. Davis and S. M. Troian, "Influence of attractive van der Waals interactions on the optimal excitations in thermocapillary-driven spreading," Phys. Rev. E 67, 016308 (2003).

${ }^{18}$ J. M. Davis, B. J. Fischer, and S. M. Troian, "A general approach to the linear stability of thin spreading films," in Interfacial Fluid Dynamics in Physiochemical Phenomena, Lecture Notes in Physics, edited by R. Narayanan (Springer-Verlag, New York, 2003).

${ }^{19}$ L. N. Trefethen, "Pseudospectra of linear operators," SIAM Rev. 39, 383 (1997).

${ }^{20}$ T. G. Wright and L. N. Trefethen, "Large-scale computation of pseudospectra using ARPACK and eigs," SIAM J. Sci. Comput. (USA) 23, 591 (2001).

${ }^{21}$ Y. Ye and H.-C. Chang, "A spectral theory for fingering on a prewetted plane,” Phys. Fluids 11, 2494 (1999). 